\title{
Gray-Matter Volume Estimate Score: A Novel Semi-Automatic Method Measuring Early Ischemic Change on CT
}

\author{
Dongbeom Song, ${ }^{a}$ Kijeong Lee, ${ }^{a}$ Eun Hye Kim, ${ }^{a}$ Young Dae Kim, ${ }^{a}$ Hye Sun Lee, ${ }^{b}$ Jinkwon Kim, ${ }^{a, c}$ \\ Tae-Jin Song, ${ }^{\text {a,d }}$ Sung Soo Ahn, ${ }^{\mathrm{e}}$ Hyo Suk Nam, ${ }^{\mathrm{a}} \mathrm{Ji}$ Hoe Heo ${ }^{\mathrm{a}}$ \\ ${ }^{a}$ Department of Neurology, Yonsei University College of Medicine, Seoul, Korea \\ ${ }^{b}$ Department of Biostatistics, Yonsei University College of Medicine, Seoul, Korea \\ 'Department of Neurology, CHA Bundang Medical Center, CHA University, Seongnam, Korea \\ dDepartment of Neurology, Ewha women's University School of Medicine, Seoul, Korea \\ ${ }^{\mathrm{e}}$ Department of Radiology, Yonsei University College of Medicine, Seoul, Korea
}

Background and Purpose We developed a novel method named Gray-matter Volume Estimate Score (GRAVES), measuring early ischemic changes on Computed Tomography (CT) semi-automatically by computer software. This study aimed to compare GRAVES and Alberta Stroke Program Early CT Score (ASPECTS) with regards to outcome prediction and interrater agreement.

Methods This was a retrospective cohort study. Among consecutive patients with ischemic stroke in the anterior circulation who received intra-arterial therapy (IAT), those with a readable pretreatment CT were included. Two stroke neurologists independently measured both the GRAVES and ASPECTS. GRAVES was defined as the percentage of estimated hypodense lesion in the gray matter of the ipsilateral hemisphere. Spearman correlation analysis, receiver operating characteristic (ROC) comparison test, and intra-class correlation coefficient (ICC) comparison tests were performed between GRAVES and ASPECTS.

Results Ninety-four subjects (age: $68.7 \pm 10.3$; male: 54 [54.9\%]) were enrolled. The mean GRAVES was $9.0 \pm 8.9$ and the median ASPECTS was 8 (interquartile range, 6-9). Correlation between ASPECTS and GRAVES was good (Spearman's rank correlation coefficient, 0.642; $P<0.001)$. ROC comparison analysis showed that the predictive value of GRAVES for favorable outcome was not significantly different from that of ASPECTS (area under curve, 0.765 vs. $0.717 ; P=0.308$ ). ICC comparison analysis revealed that inter-rater agreement of GRAVES was significantly better than that of ASPECTS ( 0.978 vs. $0.895 ; P<0.001$ ).

Conclusions GRAVES had a good correlation with ASPECTS. GRAVES was as good as ASPECTS in predicting a favorable clinical outcome, but was better than ASPECTS regarding inter-rater agreement. GRAVES may be used to predict the outcome of IAT.

Keywords Acute ischemic stroke; Acute stroke imaging; Computed tomography; ASPECTS; Prediction of outcome

\author{
Correspondence: Ji Hoe Heo \\ Department of Neurology, Yonsei \\ University College of Medicine, \\ 50-1 Yonsei-ro, Seodaemoon-gu, \\ Seoul 03722, Korea \\ Tel: +82-2-2228-1605 \\ Fax: +82-2-393-0705 \\ E-mail:jhheo@yuhs.ac \\ Received: June 30, 2015 \\ Revised: August 14, 2015 \\ Accepted: August 31, 2015
}

This study was supported by a grant of the Korea Healthcare technology R\&D Project, Ministry for Health \& Welfare Affairs, Republic of Korea (HI08C2149).

The authors have no financial conflicts of interest. 


\section{Introduction}

Early ischemic changes (EIC) on Computed Tomography (CT) represent early cytotoxic edema and possible development of irreversible tissue injury. ${ }^{1,2}$ As reperfusion to irreversibly injured tissue is not beneficial and can even be harmful if it leads to hemorrhage, European Cooperative Acute Stroke Study trials have excluded patients with EIC involving more than a third of the middle cerebral artery territory on $\mathrm{CT}^{3-5}$ However, this method is not reliable even by experienced clinicians. ${ }^{6}$ Therefore, to improve the reliability of EIC measurement, the Alberta Stroke Program Early CT Score (ASPECTS), a systematic method quantifying EIC, was devised. ${ }^{7}$ ASPECTS has proved its worth in predicting outcomes of reperfusion therapy in acute ischemic stroke, ${ }^{7-12}$ and was used to exclude patients with large infarct core in the recently published trials which showed clear benefit of intra-arterial therapy (IAT).

Nevertheless, highly trained personnel are required to measure ASPECTS and there have been conflicting reports of inter-rater agreement. ${ }^{7,13-17}$ Therefore, we have developed a novel method named Gray-matter Volume Estimate Score (GRAVES), which is expected to have better reliability as it is a semi-automatic method of measuring EIC on CT using computer software. The object of this study was to compare GRAVES and ASPECTS with regards to outcome prediction and inter-rater agreement

\section{Methods}

\section{Patients}

This was a retrospective cohort study. Candidates for this study were consecutive patients with ischemic stroke in the unilateral anterior circulation who received IAT between January 2009 and May 2012, at our institute. All those with a readable baseline CT were included. The local institutional review board approved this study and granted a waiver of consent because of its retrospective nature.

\section{Reperfusion therapy protocol and follow-up}

Our reperfusion therapy and imaging protocols have been published previously. ${ }^{18,19}$ Briefly, patients presenting within 3 hours of stroke onset were treated with intravenous tissue plasminogen activator (IV tPA) (Actylase; Boehringer-Ingelheim, Ingelheim, Germany) at a standard dose of $0.9 \mathrm{mg} / \mathrm{kg}$ ( $10 \%$ as bolus then $90 \%$ as an infusion for 60 minutes) after Non-contrast Computed Tomography (NCCT). If patients showed an unsatisfactory clinical response (defined as a $<50 \%$ improvement as measured by the National Institutes of Health Stroke Scale [NIHSS]) at the end of IV tPA infusion, IAT was considered after follow-up NCCT with CT angiography. Those patients who presented between 3-6 hours of stroke onset were also considered for IAT after NCCT with CT angiography. Reperfusion was graded by the Thrombolysis in Cerebral Infarction scale. Successful reperfusion was defined as Thrombolysis in Cerebral Infarction $2 \mathrm{~b}$ or 3 . The modified Rankin Score was assessed at 3 months after stroke onset. Favorable outcome was defined as modified Rankin Score $\leq 2$ at 3 months.

\section{Imaging acquisition}

NCCT images were obtained on a multi-detector row CT system (Sensation 64; Siemens, Erlangen, Germany or LightSpeed Plus; GE Healthcare, Milwaukee, WI, USA). The sequential axial 3-mm NCCT (Sensation 64) was performed with the following parameters: $120 \mathrm{kVp}, 300 \mathrm{mAs}$, field of view of $250 \mathrm{~mm}, 1 \mathrm{~s} /$ rotation, $30 \times 0.6 \mathrm{~mm}$ collimation and a H30s medium reconstruction kernel. The sequential axial 5-mm NCCT (LightSpeed Plus) was performed with the following parameters: $120 \mathrm{kVp}$, $250 \mathrm{mAs}$, field of view of $250 \mathrm{~mm}, 0.8 \mathrm{~s} /$ rotation, $4 \times 1.25 \mathrm{~mm}$ collimation, and a smooth reconstruction kernel.

\section{NCCT analysis using GRAVES and ASPECTS}

To measure EIC, CT scans taken just before IAT were analyzed. Two stroke neurologists independently reviewed CT scans and measured GRAVES and ASPECTS blinded to clinical information except stroke side. Any discrepancy between the two readers was resolved by reaching a consensus.

GRAVES was developed based on the software platform for image analysis (Xelis; INFINITT Healthcare, Seoul, Korea). GRAVES was defined as the estimated percentage of hypodense lesion in the gray matter of symptomatic hemisphere. With an assumption that contralateral hemisphere is normal and that two hemispheres are relatively symmetric, GRAVES was measured by subtracting estimated area of normal gray matter in the symptomatic hemisphere ( $\left.\mathrm{GM}_{\text {symptomatic }}\right)$ from that in the contralateral hemisphere $\left(\mathrm{GM}_{\text {contralateral; }} \mathrm{GRAVES}=\left[\mathrm{GM}_{\text {contralateral }}-\mathrm{GM}\right.\right.$ symptomatic $\left.] /\left[\mathrm{GM}_{\text {contralateral }}\right] \times 100\right)$. Estimated area of normal gray matter was operationally defined as an area of which Hounsfield unit (HU) is between the mean $\mathrm{HU}$ of the contralateral normal caudate head $\left(\mathrm{HU}_{\text {caudate }}\right)$ and $\mathrm{HU}_{\text {caudate }}+9$. In case of an abnormal contralateral caudate head, another normal structure of the contralateral deep gray matter such as the thalamus or lentiform nucleus was selected, at the discretion of the rater. To reflect area asymmetry between the hemispheres, estimated areas of normal brain parenchyme in each hemisphere (Parenchyme symptomatic and Parenchyme contralateral $_{\text {) }}$ predefined as area of which $\mathrm{HU}$ is between 20 and 55, were used for adjustment (Adjusted $\mathrm{GM}_{\text {contralateral }}=$ $\mathrm{GM}_{\text {contralateral }} *\left[\right.$ Parenchyme symptomatic $_{\text {Parenchyme }}$ contralateral $\left.]\right)$. CT 

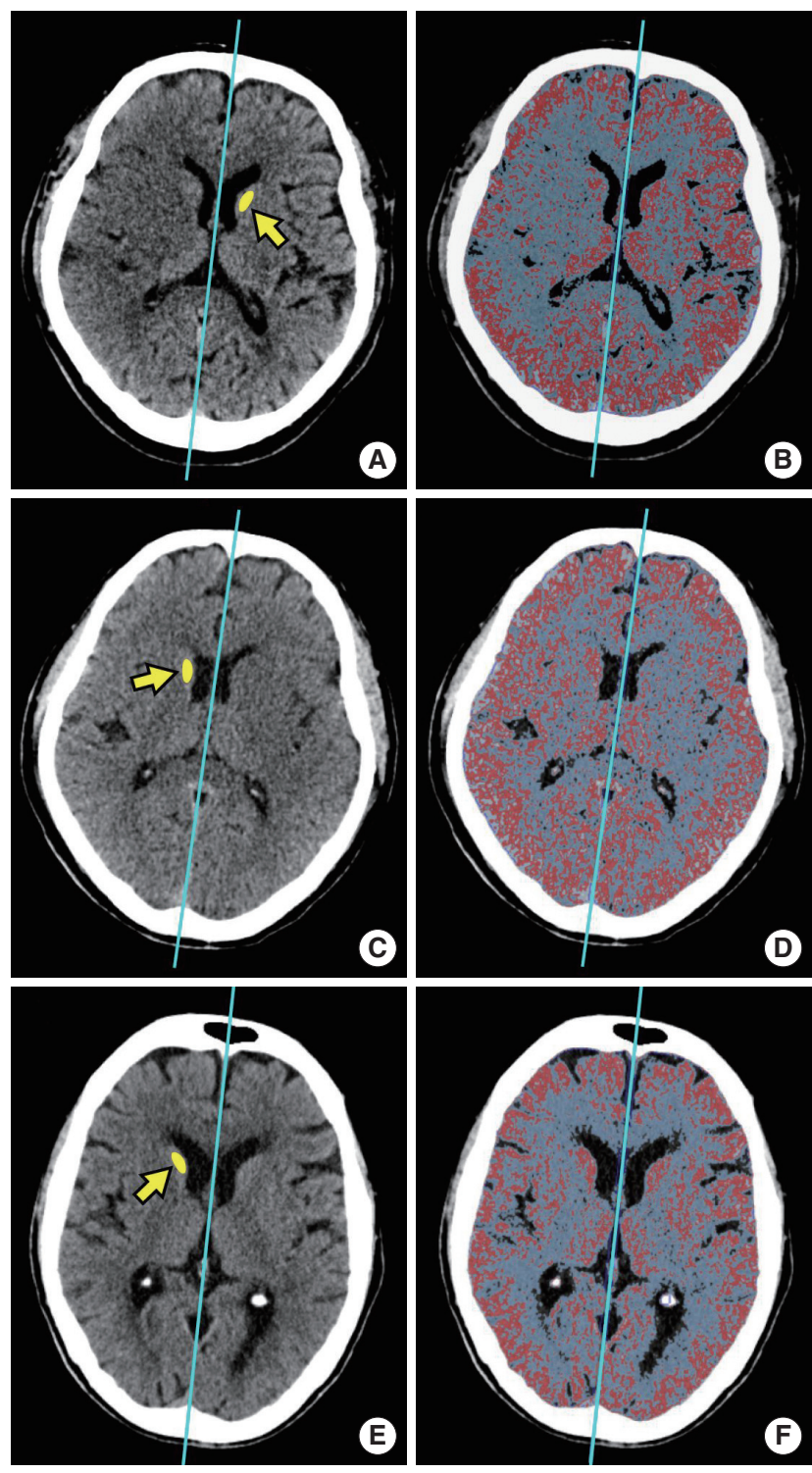

Figure 1. Representative figures of GRAVES measurements. Representative measurements of GRAVES in a patient with large core (GRAVES, 19.75; ASPECTS, 1; $A$ and B), moderate core (GRAVES, 12.93; ASPECTS, 6; C, D), and small core (GRAVES, 1.92; ASPECTS, 10; E and F) are shown. Central lines that separate the hemispheres are drawn automatically and can be adjusted by a rater. The reference values of Hounsfield unit (HU) for the gray matter are determined by drawing a region of interest on the contralateral caudate head (yellow-colored area indicated arrow; A, C, and E). Then, computer software automatically analyzes the mean $\mathrm{HU}$ of region of interest. Red colored pixels are the area of which the values of $\mathrm{HU}$ are between the mean $\mathrm{HU}$ of the contralateral caudate head $\left(\mathrm{HU}_{\text {caudate }}\right)$ and $\mathrm{HU}_{\text {caudate }}+9$. These represent estimated normal gray matter. Blue-colored pixels are the area of which the values of $\mathrm{HU}$ are between 20 and 55 . These represent estimated normal brain parenchyme (B, D, and F). GRAVES, Gray-matter Volume Estimate Score; ASPECTS, Alberta Stroke Program Early CT Score.

slices that cover $60 \mathrm{~mm}$ from upper midbrain (superior colliculus) level were all included for analysis (e.g. 20 axial slices in 3-mm NCCT, and 12 axial slices in 5-mm NCCT). To measure GRAVES, raters set the range of axial CT slices to analyze, ad- justed the central line separating the hemispheres, and drew the ROI on the contralateral caudate head. After the stated simple steps, computer software calculates GRAVES automatically and reports immediately (Figure 1).

ASPECTS was measured following recently modified definition of EIC. ${ }^{20}$ Tissue hypoattenuation and loss of gray-white matter differentiation, but not isolated cortical swelling, were taken as evidence for EIC. All axial CT images were used and settings for the window level were adjusted at the discretion of the rater to increase contrast between normal and ischemic brain areas.

\section{Statistical analysis}

Values were presented as number (\%), mean \pm standard deviation or median (interquartile range $[\mathrm{IQR}]$ ) as appropriate. Univariate analyses (independent sample t-test or Mann-Whitney $U$ test for continuous variables and $\chi^{2}$ test or Fisher's exact test for categorical variables as appropriate) were performed to compare baseline characteristics, treatment modalities, time parameters, and imaging characteristics between the favorable and the unfavorable outcome groups. Variables achieving a $P$ value less than 0.05 in the univariate analyses for favorable outcome were adjusted for the multivariate analyses (binary logistic regression models with favorable outcome as the dependent variable). Spearman correlation analysis between ASPECTS and GRAVES was performed. Receiver operating characteristic (ROC) comparison test was performed to compare predictability of a favorable outcome between ASPECTS and GRAVES. The Youden index was used to determine the optimal cut-off value of ASPECTS and GRAVES. Inter-rater agreements were assessed with linear-weighted $\kappa$ statistics, intra-class correlation coefficient (ICC) and Bland-Altman plot. ICC comparison test was performed to compare inter-rater agreement of ASPECTS and that of GRAVES. Statistical analyses were performed using the IBM SPSS Statistics for Windows Version 20.0 (IBM Corp., Armonk, NY, USA) and MedCalc (MedCalc, Mariakerke, Belgium). A two-sided $P$ value of $<0.05$ was considered statistically significant.

\section{Results}

\section{Patients enrollment}

A total of 250 patients with acute ischemic stroke received IV tPA and/or IAT at our institute between January 2009 and May 2012. Of these, 155 who received IAT were considered for inclusion in this study. Among the 155 patients, we excluded 25 with posterior circulation infarction, 3 with bilateral infarctions, 8 with unavailable modified Rankin Score at 3 months, 2 pa- 
tients with unavailable baseline CT and 23 with unreadable CT (12 with old territorial infarction, 10 with motion artifact, and 1 with brain tumor). Finally, 94 patients were enrolled in this study.

\section{Baseline characteristics}

Mean age of the study subjects was $68.7 \pm 10.3$ years and 54 (57.4\%) were men. The median NIHSS score at admission was 16 (IQR, 12-20). The median GRAVES of all subjects, internal

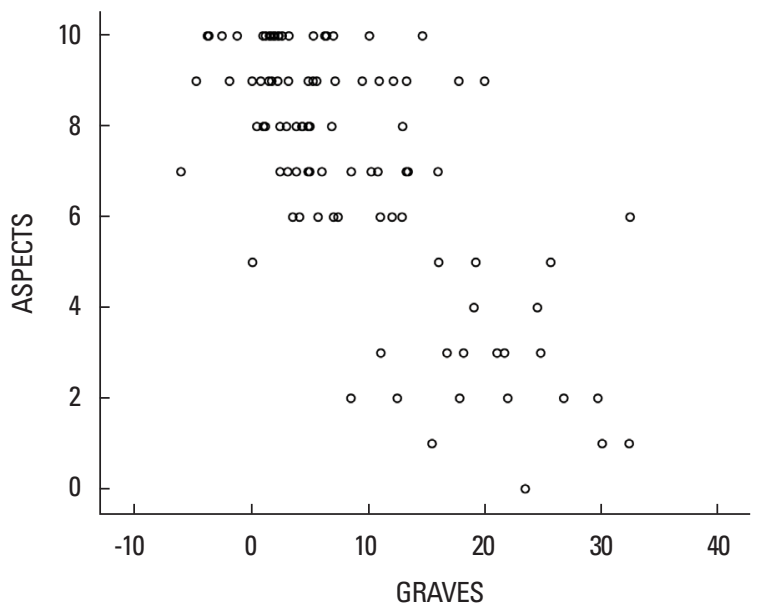

Figure 2. Correlation between the GRAVES and ASPECTS. carotid artery occlusion, middle cerebral artery M1 occlusion, and M2 occlusion was 6.2 (IQR, 2.4-13.8), 8.6 (IQR, 2.415.5), 6.1 (IQR, 2.5-16.0), and 5.0 (IQR, -0.5-9.5), respectively. The median ASPECTS of all subjects, internal carotid artery occlusion, middle cerebral artery M1 occlusion, and middle cerebral artery M2 occlusion was 8 (IQR, 6-9), 7 (IQR, 3-9), 8 (IQR, 6-10), and 9 (IQR, 7-10), respectively. We treated 58 (61.2\%) patients with combined IV tPA and IAT, and 36 (38.8\%) with IAT only. Successful reperfusion was achieved in $59(62.8 \%)$ patients, and $44(48.9 \%)$ had a favorable outcome.

\section{Correlation between GRAVES and ASPECTS}

Correlation between GRAVES and ASPECTS was good (Spearman's rank correlation coefficient, -0.642; $P<0.001$; Figure 2).

\section{Outcome prediction of GRAVES and ASPECTS}

The favorable outcome group had significantly less hypertension, less atrial fibrillation, lower NIHSS, more initial M2 occlusion, more initial IAT with Solitaire, more successful reperfusion, lower GRAVES, and higher ASPECTS compared to the unfavorable outcome group in the univariate analyses (Table 1). In the multivariate analysis adjusting for hypertension, atrial fibrillation, NIHSS, initial occlusion site, initial IAT modality and suc-

Table 1. Baseline characteristics and factors associated with favorable outcome

\begin{tabular}{|c|c|c|c|c|}
\hline & Total $(\mathrm{n}=94)$ & Favorable outcome $(n=44)$ & Unfavorable outcome $(n=50)$ & $P$ \\
\hline Sex (male) & $54(57.4)$ & $29(65.9)$ & $25(50.0)$ & 0.120 \\
\hline Age (year) & $68.7 \pm 10.3$ & $68.0 \pm 9.3$ & $69.4 \pm 11.1$ & 0.498 \\
\hline Previous cerebrovascular accident & $39(41.5)$ & $18(40.9)$ & $21(42.0)$ & 0.915 \\
\hline Diabetes Mellitus & $27(28.7)$ & $13(29.5)$ & $14(28.0)$ & 0.869 \\
\hline Hypertension & $58(61.7)$ & $21(47.7)$ & $37(74.0)$ & 0.009 \\
\hline Hypercholesterolemia & $41(43.6)$ & $19(43.2)$ & $22(44.0)$ & 0.936 \\
\hline Smoker & $15(16.0)$ & $8(18.2)$ & $7(14.0)$ & 0.581 \\
\hline Atrial fibrillation & $46(48.9)$ & $15(34.1)$ & $31(62.0)$ & 0.007 \\
\hline Initial NIHSS & $16(12-20)$ & $13(11-18)$ & $18(14-21)$ & 0.001 \\
\hline Occlusion site & & & & 0.021 \\
\hline Internal carotid artery & $43(45.9)$ & $16(36.4)$ & $27(55.0)$ & \\
\hline Middle cerebral artery M1 & $34(36.2)$ & $15(34.1)$ & $19(38.0)$ & \\
\hline Middle cerebral artery M2 & $17(18.1)$ & $13(29.5)$ & $4(8.0)$ & \\
\hline IV tPA & $58(61.2)$ & $30(68.2)$ & $28(56.0)$ & 0.225 \\
\hline Intra-arterial therapy (IAT) modality & & & & 0.012 \\
\hline Urokinase & $46(48.9)$ & $19(43.2)$ & $27(54.0)$ & \\
\hline Penumbra & $7(7.4)$ & $0(0.0)$ & $7(14.0)$ & \\
\hline Solitaire & $36(38.3)$ & $23(52.3)$ & $13(26.0)$ & \\
\hline Other & $5(5.3)$ & $2(4.5)$ & $3(6.0)$ & \\
\hline Successful reperfusion* & $59(62.8)$ & $34(77.3)$ & $25(50.0)$ & 0.006 \\
\hline Alberta Stroke Program Early CT Score (ASPECTS) & $8(6-9)$ & $9(7-10)$ & $6(3-9)$ & $<0.001$ \\
\hline Gray-matter Volume Estimate Score (GRAVES) & $6.2(2.4-13.8)$ & $3.9(1.6-6.4)$ & $11.1(5.0-20.3)$ & $<0.001$ \\
\hline
\end{tabular}

Data are presented with number $(\%)$, mean \pm standard deviation, or median (interquartile range [IQR]).

*Successful reperfusion was defined as Thrombolysis in Cerebral Infarction grade $2 \mathrm{~b}$ or 3.

NIHSS, National Institutes of Health Stroke Scale; IV tPA, intravenous tissue plasminogen activator. 
cessful reperfusion, not only ASPECTS (Odds Ratio [OR], 1.401; 95\% Confidence Interval [CI], 1.070-1.833; $P=0.014$ ), but also GRAVES (OR, 0.878; 95\% CI, 0.795-0.970; $P=0.011$ ) were independently associated with a favorable outcome.

ROC comparison analysis showed that the predictive value of GRAVES for favorable outcome (area under curve [AUC], 0.765 ; 95\% CI, 0.666-0.846) was not significantly different from that of ASPECTS (AUC, 0.717; 95\% CI, 0.615-0.805; $P=0.308$; Figure $3 \mathrm{~A})$. The optimal cutoff value of ASPECTS to predict a favorable outcome was $>7$ (Youden index, 0.37; Sensitivity, 72.7\%; Specificity, 64.0\%), while that of GRAVES was $\leq 6.5$ (Youden index, 0.47; Sensitivity, 77.3\%; Specificity, $70.0 \%)$. When we performed ROC comparison analysis for only 59 patients with successful reperfusion, the predictive value of GRAVES for a favorable outcome (AUC, 0.762; 95\% CI, 0.634-0.862) was similar to that of ASPECTS (AUC, 0.744; $95 \% \mathrm{CI}, 0.613-0.848 ; P=0.776$; Figure 3B). The predictive value of a multivariate model for a favorable outcome including
GRAVES (with age, sex, hypertension, atrial fibrillation, initial occlusion site, NIHSS, Solitaire, and successful reperfusion; AUC, 0.899 ; 95\% CI, 0.819-0.951) was similar to that of a multivariate model including ASPECTS (AUC, 0.907; 95\% CI, 0.829-0.957; $P=0.552$; Figure $3 \mathrm{C}$ ).

\section{Reliability of GRAVES and ASPECTS}

Linear-weighted $\kappa$ of ASPECTS was 0.662 (95\% CI, 0.5950.728). ICC of ASPECTS was 0.895 (95\% CI, 0.822-0.935). ICC of GRAVES was 0.978 (95\% CI, 0.966-0.985). ICC comparison analysis revealed that inter-rater agreement of GRAVES was significantly better than that of ASPECTS $(P<0.001)$. Bland-Altman plot showed that the mean difference between two measures of GRAVES was 0.4 (95\% limits of agreement, -3.1-4.0) and that of ASPECTS was 0.5 (95\% limits of agreement, -2.3-3.2). There were no significant trend between the difference and the mean of two measures for both GRAVES and ASPECTS (Figure 4).
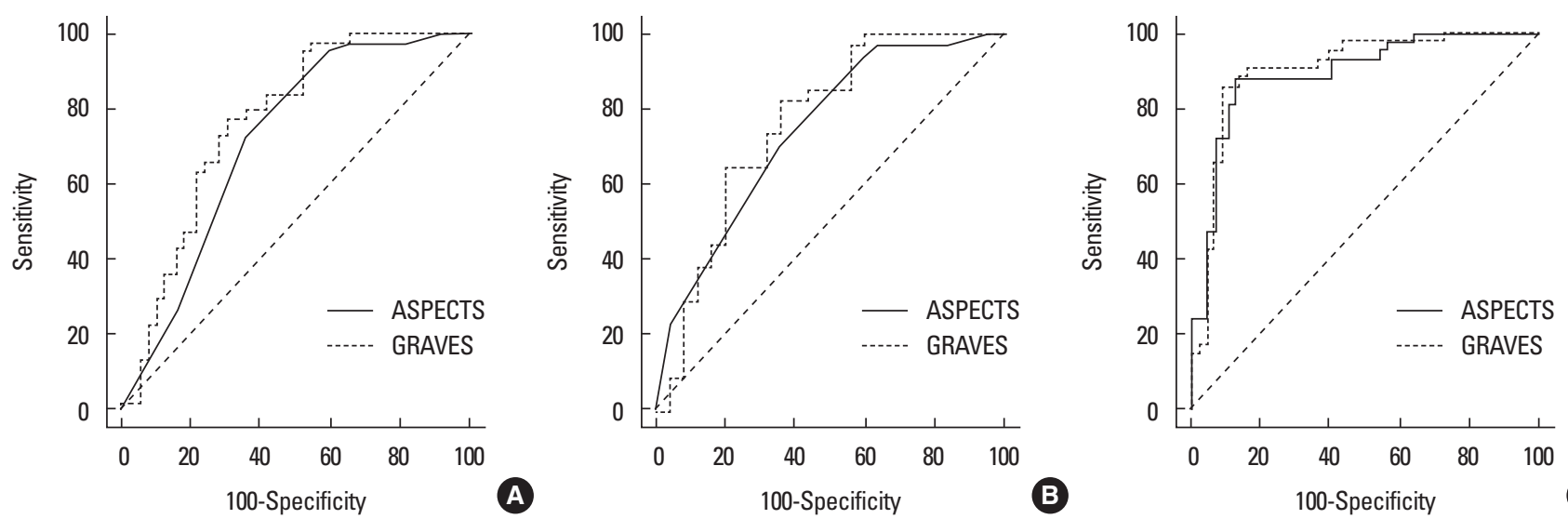

Figure 3. Receiver operating characteristic curves of the GRAVES and ASPECTS to predict favorable outcome. (A) Univariate model. (B) Univariate model with successfully reperfused patients only. (C) Multivariate model (adjusted for age, sex, hypertension, atrial fibrillation, and initial occlusion site).
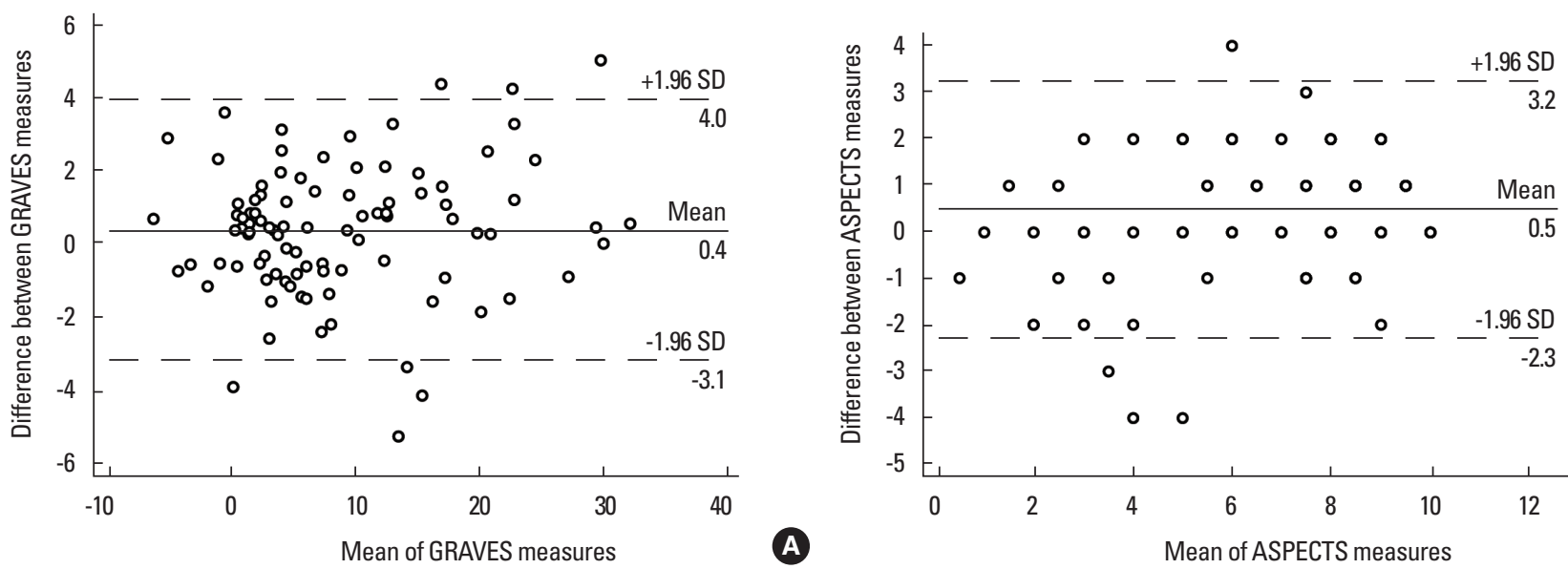

Figure 4. Bland-Altman plot demonstrating the differences between the two measures of the GRAVES and ASPECTS. Differences are plotted against the mean of two measures. The solid line indicated the mean of the difference; the dotted lines do the upper and lower 95\% limits of agreement. 


\section{Discussion}

A rapid, easy, and reliable determination of irreversibly damaged brain tissue is critical in selecting candidates for reperfusion therapy in acute stroke patients. Occlusion of the cerebral artery causes a decrease in cerebral blood flow to the downstream territory, which subsequently results in deprivation of glucose and oxygen, depletion of adenosine triphosphate, changes in ionic homeostasis, intracellular accumulation of water, and cell death in the core region of ischemia. As HU of CT is inversely correlated with the amount of water, an area of low-density lesion represents an irreversibly damaged area with cytotoxic edema. ${ }^{1,2}$ Irreversible damage is usually more prominent in the gray matter during the very early stage of infarction because gray matter is more vulnerable to ischemic insult compared to white matter. ${ }^{21}$ Classic early CT signs detailed in the literature including loss of insular ribbon, obscuration of lentiform nucleus and loss of gray-white matter differentiation ${ }^{2}$ all imply ischemic insult of gray matter. Thus, we have devised a simple method to quantify the extent of EIC by calculating the estimated percentage of hypodense lesion in the gray matter of the symptomatic hemisphere. To reflect individual difference of density in the gray matter, we calculated mean HU of contralateral caudate head and used it as a reference value of estimated normal gray matter. While the means of $\mathrm{HU}$ in caudate head were different in each patient, the standard deviations were relatively consistent between 4 and 5. Therefore, we defined the range of reference representing normal gray matter as pixels of which $\mathrm{HU}$ are between the mean $\mathrm{HU}$ of the contralateral caudate head $\left(\mathrm{HU}_{\text {caudate }}\right)$ and $\mathrm{HU}_{\text {caudate }}+9$ which accounts for two standard deviations. We chose the caudate head because it is relatively easy to distinguish and usually not affected by bone-induced streak artifact. The area of which $\mathrm{HU}$ is within the individually defined thresholds is not exclusively limited to normal gray matter. However, the areas of which $\mathrm{HU}$ is within the thresholds outside of normal gray matter is relatively small and symmetric between the hemispheres, and we operationally define the area of which $\mathrm{HU}$ is within the thresholds as the estimated area of normal gray matter. In addition, the estimated areas of normal brain parenchyme in each hemisphere were used for adjustment because the normal volume of the bilateral gray matter could be asymmetrical. While the thresholds of the normal gray matter were defined individually, lower and upper thresholds of HU for normal brain parenchyme were predefined as 20 and 55 respectively to enable rapid measurement.

It was not surprising that GRAVES was well correlated with ASPECTS given the fundamental principle of both GRAVES and ASPECTS, which were designed to estimate the EIC in the gray matter. Outcome predictability of GRAVES was also similar to that of ASPECTS, not only in the univariate model and the multivariate model, but also in the univariate model with successfully reperfused patients. GRAVES may also be used for patient selection of reperfusion treatment as ASPECTS is. However, further external validation studies are required to use GRAVES for this purpose in clinical practice. Optimal cutoff value of ASPECTS for predicting favorable outcome was $>7$ in this study, which was consistent with previously reports. ${ }^{7}$ Although further validation is required, GRAVES $\leq 6.5$ was the optimal cutoff value for predicting favorable outcome in this study.

When calculating GRAVES with computer software, raters need only to select the range of CT slices to analyze, adjust the central line to separate the hemispheres, and draw ROI on the contralateral caudate head, which can usually be done approximately within 3 minutes. Thus, raters who have a basic understanding of brain anatomy can measure GRAVES without difficulty and inter-rater agreement of GRAVES was excellent. Compared to ASPECTS which requires certain level of expertise, GRAVES may have an advantage in this regard.

This study has several limitations. First, selection bias is unavoidable considering that this study was a retrospective analysis of an IAT cohort and the sample size was only modest. Second, GRAVES could not be measured in patients with previous infarction or other structural lesions in the contralateral gray matter because it presumes the normality of the contralateral hemisphere. It was also sensitive to motion artifact. In this study, 12 patients with old territorial infarction and 10 patients with severe motion artifact who otherwise met inclusion criteria were excluded. Third, GRAVES is only applicable to patients with anterior circulation infarction.

\section{Conclusions}

GRAVES has a good correlation with ASPECTS. GRAVES was as good as ASPECTS in predicting a favorable clinical outcome, but was better than ASPECTS with regard to inter-rater agreement in patients who received IAT. Although further validation with larger sample size date is required, GRAVES and ASPECTS might be used interchangeably or alternatively to predict outcomes and aid the selection of patients for reperfusion therapies.

\section{Acknowledgments}

The authors thank Hanyoung Kim and Chang Min Shin for their excellent technical contribution to software development. 


\section{References}

1. del Zoppo GJ, von Kummer R, Hamann GF. Ischaemic damage of brain microvessels: inherent risks for thrombolytic treatment in stroke. J Neurol Neurosurg Psychiatry 1998;65:1-9.

2. Kucinski T. Unenhanced CT and acute stroke physiology. Neuroimaging Clin N Am 2005;15:397-407, xi-xii.

3. Hacke W, Kaste M, Fieschi C, Toni D, Lesaffre E, von Kummer $\mathrm{R}$, et al. Intravenous thrombolysis with recombinant tissue plasminogen activator for acute hemispheric stroke. The European Cooperative Acute Stroke Study (ECASS). JAMA 1995;274: 1017-1025.

4. Hacke W, Kaste M, Fieschi C, von Kummer R, Davalos A, Meier $\mathrm{D}$, et al. Randomised double-blind placebo-controlled trial of thrombolytic therapy with intravenous alteplase in acute ischaemic stroke (ECASS II). Second European-Australasian Acute Stroke Study Investigators. Lancet 1998;352:1245-1251.

5. Hacke W, Kaste M, Bluhmki E, Brozman M, Dávalos A, Guidetti D, et al. Thrombolysis with alteplase 3 to 4.5 hours after acute ischemic stroke. N Engl JMed 2008;359:1317-1329.

6. Grotta JC, Chiu D, Lu M, Patel S, Levine SR, Tilley BC, et al. Agreement and variability in the interpretation of early CT changes in stroke patients qualifying for intravenous rtPA therapy. Stroke 1999;30:1528-1533.

7. Barber PA, Demchuk AM, Zhang J, Buchan AM. Validity and reliability of a quantitative computed tomography score in predicting outcome of hyperacute stroke before thrombolytic therapy. ASPECTS Study Group. Alberta Stroke Programme Early CT Score. Lancet 2000;355:1670-1674.

8. Hill MD, Rowley HA, Adler F, Eliasziw M, Furlan A, Higashida RT, et al. Selection of acute ischemic stroke patients for intra-arterial thrombolysis with pro-urokinase by using ASPECTS. Stroke 2003;34:1925-1931.

9. Hill MD, Demchuk AM, Tomsick TA, Palesch YY, Broderick JP. Using the baseline CT scan to select acute stroke patients for IV-IA therapy. AJNR Am J Neuroradiol 2006;27:1612-1616.

10. Goyal M, Menon BK, Coutts SB, Hill MD, Demchuk AM, Penumbra Pivotal Stroke Trial Investigators, Calgary Stroke Program, and the Seaman MR Research Center. Effect of baseline CT scan appearance and time to recanalization on clinical outcomes in endovascular thrombectomy of acute ischemic strokes. Stroke 2011;42:93-97.

11. Hill MD, Demchuk AM, Goyal M, Jovin TG, Foster LD, Tomsick TA, et al. Alberta Stroke Program early computed tomography score to select patients for endovascular treatment: Inter- ventional Management of Stroke (IMS)-III Trial. Stroke 2014; 45:444-449.

12. Nezu T, Koga M, Nakagawara J, Shiokawa Y, Yamagami H, Furui $\mathrm{E}$, et al. Early ischemic change on CT versus diffusion-weighted imaging for patients with stroke receiving intravenous recombinant tissue-type plasminogen activator therapy: stroke acute management with urgent risk-factor assessment and improvement (SAMURAI) rt-PA registry. Stroke 2011;42:2196-2200.

13. Mak HK, Yau KK, Khong PL, Ching AS, Cheng PW, Au-Yeung PK, et al. Hypodensity of $>1 / 3$ middle cerebral artery territory versus Alberta Stroke Programme Early CT Score (ASPECTS): comparison of two methods of quantitative evaluation of early CT changes in hyperacute ischemic stroke in the community setting. Stroke 2003;34:1194-1196.

14. Coutts SB, Demchuk AM, Barber PA, Hu WY, Simon JE, Buchan AM, et al. Interobserver variation of ASPECTS in real time. Stroke 2004;35:e103-e105.

15. Gupta AC, Schaefer PW, Chaudhry ZA, Leslie-Mazwi TM, Chandra RV, González RG, et al. Interobserver reliability of baseline noncontrast CT Alberta Stroke Program Early CT Score for intra-arterial stroke treatment selection. AJNR Am J Neuroradiol 2012;33:1046-1049.

16. Finlayson O, John V, Yeung R, Dowlatshahi D, Howard P, Zhang $\mathrm{L}$, et al. Interobserver agreement of ASPECT score distribution for noncontrast CT, CT angiography, and CT perfusion in acute stroke. Stroke 2013;44:234-236.

17. Psychogios MN, Schramm P, Frölich AM, Kallenberg K, Wasser K, Reinhardt L, et al. Alberta Stroke Program Early CT Scale evaluation of multimodal computed tomography in predicting clinical outcomes of stroke patients treated with aspiration thrombectomy. Stroke 2013;44:2188-2193.

18. Lee KY, Kim DI, Kim SH, Lee SI, Chung HW, Shim YW, et al. Sequential combination of intravenous recombinant tissue plasminogen activator and intra-arterial urokinase in acute ischemic stroke. AJNR Am J Neuroradiol 2004;25:1470-1475.

19. Song D, Kim BM, Kim DJ, Kim YD, Kim J, Lee HS, et al. Comparison of stent retriever and intra-arterial fibrinolysis in patients with acute ischaemic stroke. Eur J Neurol 2014;21:779-784.

20. Puetz V, Dzialowski I, Hill MD, Demchuk AM. The Alberta Stroke Program Early CT Score in clinical practice: what have we learned? Int J Stroke 2009;4:354-364.

21. Arakawa S, Wright PM, Koga M, Phan TG, Reutens DC, Lim I, et al. Ischemic thresholds for gray and white matter: a diffusion and perfusion magnetic resonance study. Stroke 2006;37: 1211-1216. 